Images dans le monde ibérique et ibéricoaméricain

5 | 2012

Le visible et l'invisible dans le monde hispanique et hispano-américain

\title{
Deux représentations de la vision de Saint Jean à Patmos : Le Gréco (1595) et Vélasquez (1618)
}

\section{Edgard Samper}

\section{(2) OpenEdition \\ Journals}

Édition électronique

URL : http://journals.openedition.org/agedor/854

DOI : 10.4000 /agedor.854

ISSN : 2104-3353

Éditeur

Laboratoire LISAA

Référence électronique

Edgard Samper, « Deux représentations de la vision de Saint Jean à Patmos : Le Gréco (1595) et Vélasquez (1618) », L'Âge d'or [En ligne], 5| 2012, mis en ligne le 01 mars 2012, consulté le 16 décembre 2019. URL : http://journals.openedition.org/agedor/854 ; DOI : 10.4000/agedor.854 
Edgard SAMPER

Université de Saint-Etienne

\section{DEUX REPRÉSENTATIONS DE LA VISION DE SAINT JEAN À PATMOS : LE GRÉCO (1595) ET VÉLASQUEZ (1618)}

Résumé : Il s'agit d'analyser ces deux tableaux qui représentent la même vision de saint Jean à Patmos et dont l'origine commune est le texte de l'Apocalypse. Au sein de ce cadre rigide qu'est la Contre-Réforme, les deux grands artistes ne figurent pas l'invisible de la même façon mais la dialectique entre le visible et l'invisible exprime, dans les deux cas, la transcendance et ils figurent le lieu possible de la Révélation.

Mots-clés : peinture religieuse - vision - saint Jean à Patmos - Le Gréco - Vélasquez - ContreRéforme

Resumen : Se trata de analizar los dos cuadros que representan la misma visión de san Juan en Patmos, cuyo origen similar es el texto apocalíptico. Dentro del marco relativamente rígido de la Contrarreforma, los dos grandes artistas no figuran lo invisible de la misma manera pero la dialéctica entre visible e invisible expresa estéticamente, en ambos casos, la trascendencia y figuran el lugar posible de la Revelación.

Palabras clave : pintura religiosa - visión - san Juan en Patmos - El Greco - Velázquez Contrarreforma

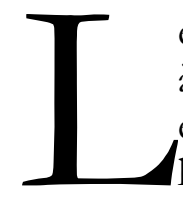

es toiles que nous nous proposons d'étudier appartiennent à ce que l'on nomme, à la Renaissance et à l'Âge classique, la peinture de vision. La Réforme catholique et les préceptes tridentins légitiment l'image et redécouvrent le rôle de l'imagination dans l'exercice de la foi. Loyola, dans sa contemplation, incite à : «voir avec la vue de son imagination ». Plus que jamais, l'art possède le pouvoir de figurer l'invisible, de donner à voir une expérience intérieure et incommunicable ${ }^{2}$.

Mais le terme même de vision reste à définir. En tant que spectateur, nous sommes confrontés à deux expressions différentes de l'immatérialité : la vue, comme perception phénoménologique et la vision, comme représentation. Pour saint Thomas d'Aquin, dans

\footnotetext{
1 Dans [47] «Meditación con las tres potencias sobre el 1, 2 y 3 pecado », texte de la version dite « autographe » des Exercices spirituels de Loyola (1554), cité dans FABRE, A. P., Ignace de Loyola, le lieu et l'image, Paris, J. Vrin, 1992, p. 28.

2 Voir, de WIRTH, Jean, «L'apparition du surnaturel dans l'art du Moyen Âge », L'image et la production du sacré, sous la direction de Françoise Dunand, Jean-Michel Spieser et Jean Wirth, Paris, Méridiens Klincksieck, 1991, p. 138-164 (p. 140). Auparavant, cf. l'ouvrage de MÂLE, Emile, El arte religioso de la Contrarreforma, Madrid, Ediciones Encuentro, (nous consultons l'édition de 2001) ainsi que celui de GALLEGO, Julián, Vision et symboles dans la peinture du siècle d'or espagnol, Paris, Klincksieck, 1968.
} 
sa Summa Theologica, le terme contient deux acceptions : la première concerne la perception organique (la vue); la seconde s'applique à la perception interne de l'imagination ou de l'intellect ${ }^{3}$. L'expérience visionnaire n'est pas nécessairement une expérience optique, bien qu'elle soit une expérience de l'image ${ }^{4}$. Quelle est la signification théologique de l'apparition et de la vision ${ }^{5}$ ? Au sens le plus large, ce sont des expériences spirituelles exceptionnelles qui rendent accessibles aux sens corporels les réalités invisibles comme Dieu, les anges, les saints, et le tout dans un but eschatologique. Depuis le temps du christianisme oriental, l'icône qui figure au cœur de l'expérience spirituelle est bien le reflet visible de l'invisible, une «image» au sens théologique du terme avant même d'être une image plastique. Depuis, l'œuvre d'art et la peinture en particulier, donc le visible par excellence, sont devenues un élément médiateur privilégié entre un visible et un invisible, traduits, comme nous le verrons pour Le Gréco et Vélasquez, par des solutions picturales figuratives bien différentes ${ }^{6}$. En 1649, Pacheco, théoricien de l'art sacré, et surtout Carducho, en 1663, sont les auteurs d'un débat élaboré sur les solutions pratiques relatives à l'élaboration de l'image de vision ${ }^{7}$. Vision, dans sa dualité, à la fois comme acte de voir et comme contenu visuel.

Les deux peintres espagnols transmuent donc en visible, pour des spectateurs potentiels, ce qui fût, sans eux, demeuré invisible. Nous tâcherons de saisir quelle est la viabilité de l'expression «montrer l'invisible». Nous analyserons le sens de ces toiles en fonction, d'une part, du cadre réglé par le Concile de Trente et de la source narrative et doctrinale du thème iconographique de Saint Jean à Patmos et, d'autre part, nous utiliserons modestement la philosophie de l'art de Merleau-Ponty et de certains de ses disciples dans la mesure où la phénoménologie s'oriente vers une iconographie « de la vision ». Les travaux de Jean-Luc Marion établissent une différence entre invisible et « invu» dans le domaine de l'esthétique. Cette notion, d'une grande force heuristique, nous aidera ainsi que les écrits de Herman Parret sur les Épiphanies de la présence et ceux de Georges Didi-Huberman qui distingue le visuel et le visible ${ }^{8}$.

La source se trouve dans l'Apocalypse johannique (I, 9-20), texte fondateur de la chrétienté qui vient ponctuer le Nouveau Testament. Jean est envoyé en exil sur un îlot du Dodécanèse par l'empereur Domitien, après avoir subi le supplice de l'huile bouillante, duquel il est sorti indemne. Il y aurait rédigé l'Apocalypse, l'une de ses nombreuses visions allégoriques et prophétiques ; c'est ainsi qu'il sera plus tard souvent représenté, accompagné de l'Aigle, emblème de force et de majesté dans la tradition du Tétramorphe. Dans le

\footnotetext{
3 Summa Theologica, I, q. LXVII, a, 1, cité par STOICHITA, Victor I., dans son introduction, El ojo mistico. Pintura y visión en el Siglo de Oro español, Madrid, Alianza Forma, 1996, p. 11.

${ }^{4}$ Voir l'excellente approche iconographique de l'image de vision par STOICHITA, Victor I., ibid.

${ }^{5}$ Pour la différence entre apparition et vision, voir l'article de FABRE, Pierre-Antoine, « Les Visions d'Ignace de Loyola dans la diffusion de l'art jésuite », vol. 114, N4, French Issue, The Johns Hopkins University Press (Septembre, 1999), p. 816-847. A la p. 838, l’auteur écrit : « la vision nous est montrée à nous, qui regardons le voyant, alors que l'apparition apparait de fait à celui auquel, dans l'espace fictif de la représentation, elle s'adresse puisqu'aussi bien elle n'apparaît qu'à lui ».

${ }^{6}$ Pour ce mode d'accès à l'invisible, voir l'article de COULOUBARITSIS, Lambros : "Le mythe et l'art comme modes d'accès à l'invisible » p. 8-22 dans les Actes du colloque de 1992 : Montrer l'invisible. Figuration et invention du réel dans la peinture, sous la direction de Jean-Pierre Sylvestre, Dijon, collection Figures libres, 1994. ${ }^{7}$ Dans son Arte de la Pintura, imprimé en 1649, Pacheco affirme : «los cuerpos de pensamiento son los que la imaginación forma », dans l'édition de B. Bassegoda i Hugas, Madrid, 1999, p. 73 et, pour Carducho : Diálogos de la Pintura. Su defensa, origen, esencia, definición, modos y diferencias, (1663), édition de F. Calvo Serraller, Madrid.

8 MARION, Jean-Luc, L'Idole et la distance, Paris, Grasset, 1977 et La Croisée du visible, Paris, Editions de la Différence, 1991, réed. PUF, 2001. PARRET, Herman, Epiphanies de la présence, Limoges, Pulim, 2006, et DIDI-HUBERMAN, Georges, Devant l'image, Paris, Minuit, 1990.
} 
chapitre I, l'évangéliste explique qu'il eut la vision du Christ et que ce dernier lui dit: "Ce que tu vois, écris-le dans un livre ». L'Apocalypse est un ouvrage de visions et l'auteur ne cesse d'affirmer qu'il « voit» et qu'il doit consigner ce qu'il «voit» pour les églises d'Asie. Le mot «apocalypse » est la transcription d'un terme grec signifiant mise à nu, enlèvement du voile ou révélation. Cette révélation, basée sur des visions, est donc un dévoilement permis par des signes qui restent à déchiffrer. Dans le douzième chapitre, deux signes apparaissent dans le ciel à l'Apôtre qui voit, entend et rédige sur terre : la Femme, élément central de l'Apocalypse, nouvelle Ève qui annonce le renouveau de l'Homme $(12,1)$, et le dragon finalement vaincu $(12,3)$. Nous sommes donc, dès le point de départ, en présence d'une dichotomie spatiale et d'un passage de la vision à la parole et de la parole à l'écriture. Dans les deux toiles étudiées, Jean dirige son regard vers la partie supérieure de la composition : il y a simultanéité d'une scène « réelle » et d'une vision miraculeuse. Le Gréco et Vélasquez sont les héritiers d'une longue tradition manuscrite et iconographique car, dès le Moyen Âge, l'Apocalypse s'échappe du livre pour envahir l'espace visuel sur de multiples supports : sculptures, vitraux, enluminures (Les Très Riches Heures du Duc de Berry, les Beatus'), tapisseries (Angers), gravures et peintures.

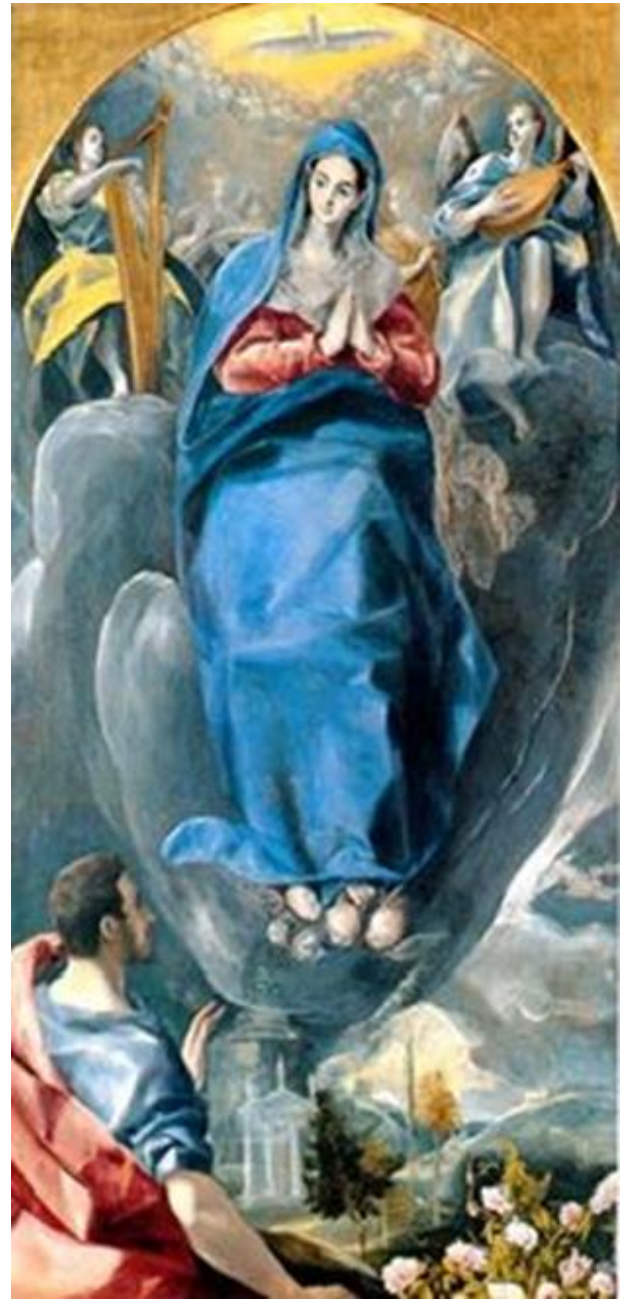

Le Gréco, Saint Jean à Patmos, musée de Santa Cruz de Tolède (1580, 236 x 138).

\footnotetext{
9 Par exemple, dans le célèbre Beatus de Liebana. Voir STIERLIN, Henri, « Le Livre de Feu ». L'Apocalypse et l'art mozarabe, Genève, Sigma, 1978.
} 
Commençons par la représentation du Gréco du musée de Santa Cruz de Tolède (1580, 236 x 138). Jean, le sujet voyant, occupe le premier plan de l'angle inférieur gauche ; ce qui indique clairement le point à partir duquel un œil occidental doit commencer la lecture. Il est peint à mi-corps, comme spectateur intégré dans un registre terrestre: Patmos, lieu défini de l'écriture. Nous sommes bien loin de l'évangéliste surdimensionné et stupéfait de l'Ouverture du cinquième Sceau, autre toile apocalyptique du Metropolitan Museum de New York. On ne retrouve pas le vaste décor végétal et animal qui entoure le saint et forme une sorte de cadre autour de lui comme la tradition semblait l'imposer dans le retable de Saint Jean à Patmos de Hans Burgkmair (1518, huile sur bois, Alte Pinakothek, Munich) ou la toile plus célèbre de Jérôme Bosch (1504-5, Gemäldgalerie, Berlin). Le paysage de cet artiste visionnaire et inventeur d'un monde fantastique, marque encore l'éloignement relatif des plans par des transitions colorées, chères aux primitifs. Le peintre candiote, lui, organise une chorégraphie majestueuse qui se déploie devant le spectateur, aussi bien Jean que nous-mêmes. Dans ce «dédoublement iconique " ${ }^{10}$, composante nécessaire de tout tableau de vision, au-dessus de l'apôtre, surgit d'entre les nuages le corps glorieux et monumental de la Vierge, entouré d'un dispositif scénographique vertigineux. Le système de proportions que Le Gréco utilise relève d'une hiérarchie spirituelle sans aucun rapport avec la logique des arpenteurs. Dans une étude sur la diffusion de l'art jésuite, Pierre-Antoine Fabre souligne ces changements d'échelle inhérents à toute peinture représentant une vision : "la vision - dit-il - phénomène interne, est en quelque sorte aux dimensions de l'âme, c'est-à-dire sans nulle mesure matérielle »11. La verticalité de l'expérience théophanique nait d'une expérience suprasensorielle, impensable sans une mise en scène active du corps voyant et répond aux impératifs d'une double rhétorique contreréformiste : celle de la communication avec le sacré et celle de la transmission de cette même expérience au spectateur qui participe par empathie à l'acte de vision. C'est grâce à la prédominance de cet axe vertical, lié à une dynamique de l'imagination ascensionnelle, que s'opère le passage entre la terre et le ciel, le visible et l'invisible ${ }^{12}$. Ce ciel s'entrouvre et laisse voir, presque entendre, le concert angélique. L'un des anges adolescents regarde avec douceur vers le Saint-Esprit, l'autre se concentre sur l'instrument de musique. Ils connaissent tous deux les mystères qui nous échappent. Cette omniprésence des anges a été renforcée par la littérature apocryphe concernant la Vierge; depuis l'Annonciation jusqu'au terme de son expérience, elle est emportée au ciel par les anges ${ }^{13}$. Ce que Hubert Damisch nomme "la nuée mystique ", sorte de mandorle de nuages, la soustrait aux lois de la pesanteur et «manifeste l'ouverture de l'espace profane sur un autre espace, qui lui donne sa vérité $»^{14}$. Marie semble flotter, avancer dans les airs, dans un halo de lumière presque insoutenable et qui émane d'elle, rappelant ainsi le texte ignacien, bien connu du Gréco : «le parecía ver una cosa blanca; de la qual salían algunos rayos, y que della hacía Dios lumbre $\gg^{15}$. Le peintre tolédan, en dessinant les contours physiques d'êtres de lumière, matérialise l'immatériel et montre comment le mystère d'une présence invisible s'incarne

\footnotetext{
${ }^{10}$ L'expression est empruntée à STOICHITA, Victor I., op. cit., p. 29.

11 «Art. cit. », p. 816.

${ }^{12}$ Cf., RIBON, Michel, A la recherche du temps vertical dans l'art. Essai d'esthétique, Paris, Kimé, 2002, p. 19.

13 Voir l'article de CASSAGNES BROUQUET, Sophie, "L'univers des créatures célestes. Premières apparitions des anges et des démons dans l'art occidental ", Montrer l'invisible. Figuration et invention du réel dans la peinture, sous la direction de Jean-Pierre Sylvestre, Actes du colloque des 18-22 mai 1992, Dijon, Collection Figures libres, 1994, p. 50-72.

${ }^{14}$ DAMISCH, Hubert, Théorie du nuage. Pour une histoire de la peinture, Paris, Seuil, 1972, p. 65-66.

${ }^{15}$ Cf. l'article cité de Pierre-Antoine Fabre, «Les Visions d'Ignace de Loyola... » et l'importance de la lumière, p. 822. MURRAY, L., La Haute Renaissance et le maniérisme, Paris, Thames et Hudson, 1995, p. 275, précise que Le Gréco connaissait fort bien les Exercices spirituels.
} 
dans la visibilité. La brillance caractéristique des innombrables drapés, obtenue par l'application d'un glacis à l'huile, riche et translucide et d'un pigment à base d'azurite fait vaciller le spectateur entre la présence intensément charnelle et le propos théologique.

Le Crétois, en réalité, abandonne l'attirail traditionnel du thème de Saint Jean à Patmos (aigle, dragon, paysage marin, faune et flore abondantes) au profit d'une dynamique instaurée par la composition, la gestuelle des figures et les regards. Si l'on compare cette représentation à celle d'autres peintres espagnols, comme Juan Sánchez Cotán ${ }^{16}$, Pedro de Orrente $^{17}$ ou même Vélasquez, l'évangéliste du Gréco qui avait été et serait la figure centrale des toiles, disparait presque, écrasé par la vision de la Vierge entourée d'anges et de nuages. Que contemple donc l'apocalypticien? Une image hybride de la madone tota pulchra, à la fois la Femme de l'Apocalypse, personnifiant l'Eglise, la Vierge de l'Assomption élevée vivante par les anges jusqu'au paradis, fidèle aux récits de La Légende dorée, et l'Immaculée Conception. L'identité de cette dernière est clairement explicitée par les symboles immaculistes des Litanies dans le paysage de la partie inférieure de la composition (tour fortifiée de David, fontaine d'eau pure, roses, lys...) où se confondent Patmos et la Jérusalem céleste. Que représente Le Gréco ? La vision de Jean à Patmos ou, comme le suggère Stoichitia lorsqu'il parle d'une méditation métapicturale ${ }^{18}$, l'évangéliste en train de contempler un tableau céleste, une image peinte par le pinceau divin (le Deus Pictor), comme l'on considérait à l'époque la figure de l'Immaculée ?

Georges Didi-Huberman distingue le visuel et le visible. Face à l'œil - explique-t-il -il n'y a que le visible, le règne de l'optique ; le visuel concerne davantage le regard, ce qui signifie que l'on doit tenir compte, en analysant les toiles du Gréco et de Vélasquez, d'une certaine fragilité, au moins d'une mouvance de la perception. L'esthéticien ajoute: «l'invisible est l'horizon du visuel ${ }^{19}$. À cette démarche, nous préférons la théorie de JeanLuc Marion qui introduit la notion de l'invu: «l'invu relève certes de l'invisible, mais ne se confond pas avec lui, puisqu'il peut le transgresser en devenant précisément visible [...]. Le peintre est celui qui filtre l'accès de l'invu au visible $»^{20}$. Dans le catholicisme, cette dialectique du visible (ou de l'invu) et de l'invisible, particulièrement dans le cas de Saint Jean à Patmos, repose sur le principe même de l'incarnation comme présence. Le Verbe (Logos) s'est fait chair et s'est donc rendu visible; la difficulté liée au caractère non accessible du modèle divin s'efface lorsque l'invisible prend corps. "C'est parce que vous dites je vois que vous êtes aveugles. Je suis venu pour que ceux qui ne voient pas voient», déclare Jésus, dans L'Évangile selon saint Jean (9, 39).

\footnotetext{
${ }^{16}$ Huile sur toile, 67 x 71, Musée de Santa Cruz, Tolède.

17 Huile sur toile, 99 x 131, Prado. Voir aussi une peinture sur bois de Diego de Aguilar, 1600, 37 x 25, couvent de Saint Dominique de Tolède (intérieur de la porte d'un retable). À ce sujet, cf. Diego Angulo Iñiguez, PÉREZ SÁNCHEZ, Alfonso, Pintura toledana de la primera mitad del siglo XVII, Madrid, Instituto Diego Velázquez, CSIC, 1972, et PÉREZ SÁNCHEZ, Alfonso, Pintura barroca en España (1600-1750), Madrid, Cátedra, 1992.

18 Ibid.

${ }^{19}$ Dans Devant l'image, Paris, Minuit, 1990, p. 21-64.

${ }^{20}$ La Croisée du visible, Paris, Éditions de la Différence, 1991 réed. PUF, 2001, p. 51-52.
} 


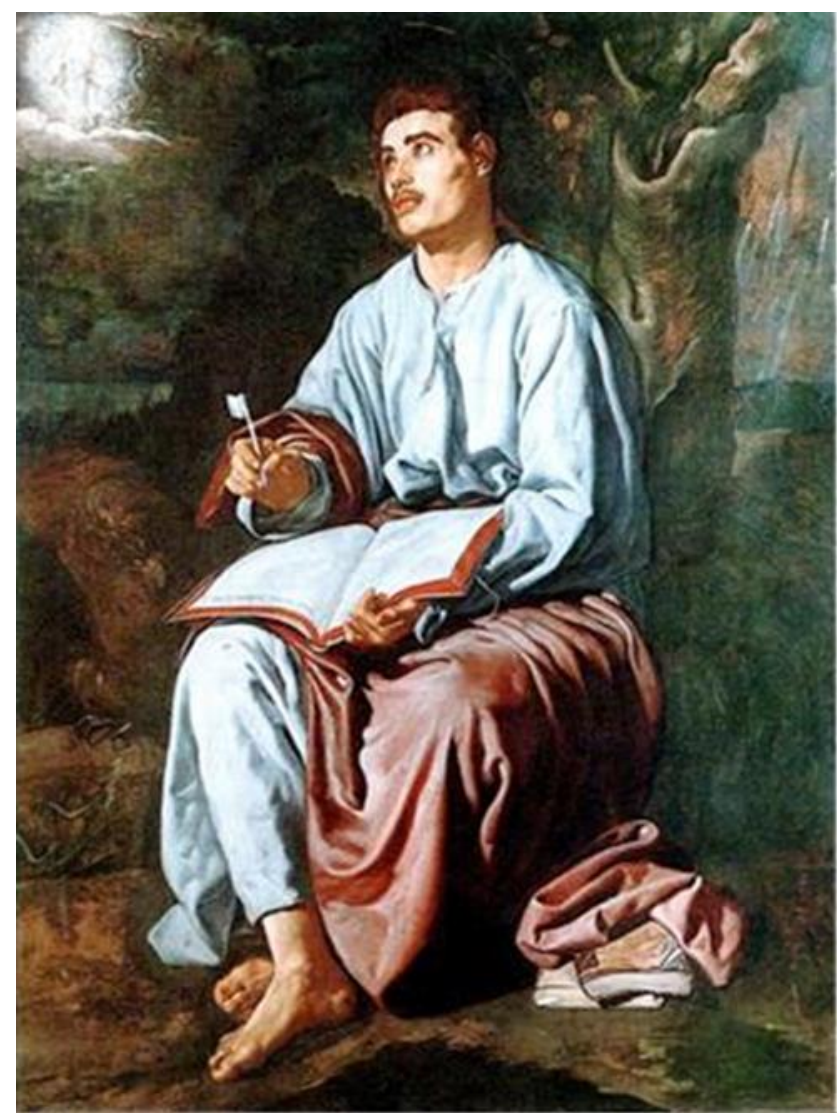

Velázquez, Saint Jean à Patmos, National Gallery, Londres (1619, 135 x 102).

Dans la toile de Vélasquez (1619, 135 x 102, Londres, National Gallery), œuvre de jeunesse, notre attention se centre sur la figure du jeune apôtre qui apparait assis ; le livre dans lequel il écrit le contenu de la révélation posé sur les genoux. À ses pieds, deux autres livres évoquent l'évangile et les trois épîtres dont il est l'auteur. Le visage du saint a été peint d'après nature. Il s'agirait du même modèle que celui de la tête de profil du Musée de l'Ermitage. Le visage rude et décharné, les pommettes hautes, les lèvres épaisses, la moustache et les sourcils fournis ne font aucune concession aux critères d'idéalisation. En le dessinant ainsi, sans doute, Vélasquez a voulu transposer sur la toile un des conseils iconographiques de son maitre Pacheco, qui recommandait de ne pas peindre Jean comme un vieillard, mais sous les traits «d'un jeune homme, à cause de sa virginité et pour proposer à chacun un modèle de pureté, attaché à consacrer au Christ la fleur de sa jeunesse $»^{21}$. Dans la pénombre se tient l'aigle. Sa présence se devine grâce à une des serres ainsi qu'à quelques taches blanches sur le bec et la tête. Le plumage demeure confondu avec la gamme terreuse et monochrome du paysage. À la droite de l'arbre, la claire-voie est estompée par des touches pâteuses, caractéristiques de Vélasquez. Ce paysage projette le modèle vers l'avant et la lumière puissante, issue d'un point situé hors de la toile, accentue l'architecture du visage et souligne, par des ombres épaisses, le volume et les plis du drapé. Cette étude très minutieuse de la lumière sur cette puissante figure masculine fait ressortir encore plus le caractère surnaturel de la vision, enveloppée dans une aura de lumière diffuse. Dans l'angle gauche supérieur apparaît le contenu de la vision qu'a eue le saint, (Apocalypse, 12, 1-4), la Femme de l'Apocalypse, interprétée plus tard comme l'Immaculée

${ }^{21}$ El Arte de la pintura, éd. de Francisco Bonaventura Bassegoda Pacheco, Madrid, Cátedra, 1990, p. 673. 
Conception, dont la définition dogmatique controversée avait à Séville d'ardents défenseurs :

Un grand signe parut dans le ciel : une femme enveloppée du soleil, la lune sous ses pieds, et une couronne de douze étoiles sur sa tête [...]. Un autre signe parut encore dans le ciel; et voici, c'était un grand dragon rouge, ayant sept têtes et dix cornes, et sur ses têtes sept diadèmes.

Ainsi était-elle déjà présente dans la partie centrale du retable de Hans Baldung (1511, Metropolitan Museum of Art, New York) et dans le volet droit du triptyque de Memling (1474-79, hôpital Saint-Jean de Bruges). Minuscule, se détachant sur un ciel d'azur, la Femme, dans sa mandorle, terrasse le dragon qui tente de s'attaquer à elle. Dans les toiles espagnoles de Sánchez Cotán et Pedro de Orrente, représentant la vision de Patmos, le spectateur retrouve cette image, à droite de la composition, toujours plus visible que dans la toile de Vélasquez, excepté le cas de Alonso Cano (1646-50, 218 x 153, Musée des BeauxArts de Budapest).

La vision et l'écriture sont liées dans l'expérience de Jean et, chez Vélasquez, le geste suspendu de l'apôtre est éloquent : il interrompt la rédaction de ce livre à la fois descriptif et prospectif et, touché par la grâce, il oriente son regard vers les rayons de lumière divine. Ce que nous voyons, nous, c'est l'acte même de vision. Le contenu demeure imperceptible, à peine déchiffrable et se soustrait au regard humain. L'effusion irradiante et éblouissante de la nuée semble déchirer le ciel ténébreux. Pour Vélasquez, dans cette toile, l'usage de la lumière ne confirme plus les formes visibles comme dans ses premières toiles caravagesques, mais, au contraire, les confond, les dématérialise et les dissout au profit de l'apparition de l'invisible. Ce qu'Aristote nommait « le diaphane lumineux », crée une sorte d'intervalle, d'intermédiaire, de mi-lieu et le jeu de l'évanescence souligne le phénomène de transcendance tout en montrant à notre regard ses propres limites ${ }^{22}$. L'invisibilité ou l'invu est ici l'informe. Dès lors, comment ne pas penser à l'ouvrage posthume de Merleau-Ponty, Le visible et l'invisible (1964) ${ }^{23}$. Pour le philosophe français, la question du sens ne s’inscrit pas dans une ontologie dualiste de l'apparence et de l'être. Il existe une réversibilité du visible et de l'invisible ; l'invisible n'étant pas l'opposé du visible. Bien au contraire, c'est l'invisible qui donne ses possibilités au visible, qui l'habite, le creuse en profondeur. Il en est la puissance. Cette cohabitation (visible/invisible, corps/esprit, présence/absence) est, selon Merleau-Ponty, un signe de l'ouverture de l'Homme à la transcendance.

\footnotetext{
${ }^{22}$ Selon la définition dans le traité d'ARISTOTE, De l'Ame, trad. par E. Barbottin, Paris, Les Belles Lettres, 1989. Voir, à ce sujet VASILIU, Anca, Du diaphane. Études de philosophie médiévale, Paris, Librairie Vrin, 1997 et DIDI-HUBERMAN, Georges, Phasmes, essais sur l'apparition, Paris, éd. De Minuit, 1998.

${ }^{23}$ Texte établi par LEFORT, Claude, Paris, Éditions Gallimard, collection « Bibliothèque des Idées », 1964.
} 


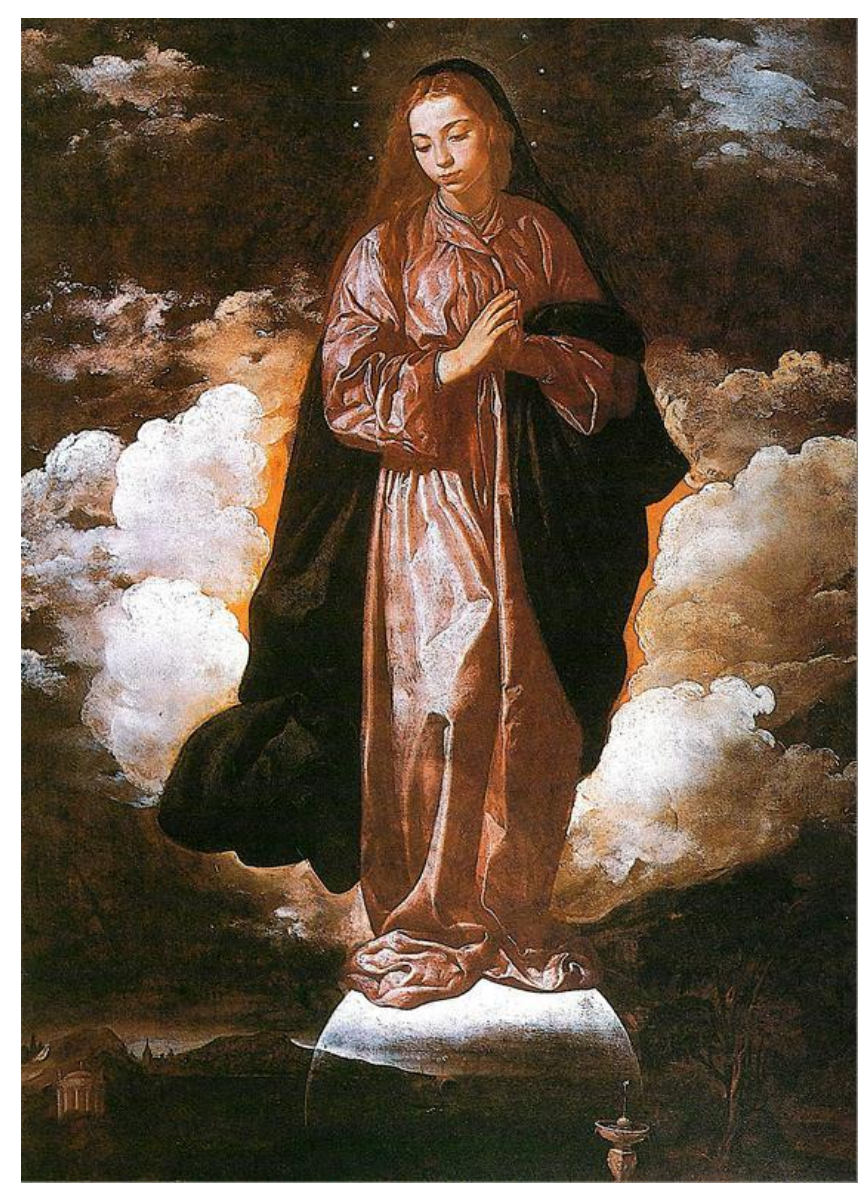

L'Immaculée Conception (1618, 135 x 102, Londres, National Gallery).

Il nous faut voir aussi, dans le tableau du maitre sévillan, une spéculation autour du thème métapictural de l'Immaculée Conception. Si Vélasquez réduit ainsi le contenu de la vision de Saint Jean à une forme presque invisible, c'est sans doute qu'il garde, pour une autre toile, la totale apparition de Patmos. Comme nous l'avons déjà signalé, Jean a une plume dans la main et, dans le livre qu'il tient sur ses genoux, on distingue quelques lignes sur la page de droite, mais celle de gauche reste vierge. Nous pensons, après Victor Stoichita, que la clé explicative se trouve dans L'Immaculée Conception (1618, 135 x 102, Londres, National Gallery), de dimensions identiques et qui provient également de la salle capitulaire du couvent des Carmes à Séville, qui serait une sorte de pendant ou de diptyque ${ }^{24}$. Stoichita écrit : « el cuadro de la Inmaculada Concepción viene, por así decirlo, a llenar el vacío de la página en blanco sobre la que el Evangelista ha podido tan sólo escribir dos líneas » ${ }^{25}$. Marie prend les traits de la Femme de l'Apocalypse et de l'Immaculée ; elle s'élève en majesté selon un traitement iconographique orthodoxe, fixé par Pacheco et adopté par son disciple : la Vierge porte une robe teintée de magenta avec des plis profonds. Partiellement entourée de nuages blancs opaques, elle est auréolée de douze étoiles. On retrouve, dans le paysage de la partie basse, les symboles des litanies déjà précisées pour la toile du Gréco. Ce n'est pas un hasard si les deux toiles de Vélasquez ont été peintes quelques années après la création, en 1616, de la Real Junta de la Inmaculada Concepción, dogme qui instaurait une relation étroite entre la Femme de la vision de saint Jean à Patmos et l'Immaculée, Reine

24 Voir BROWN, Jonathan, Velázquez, Paris, Fayard, 1988, p. 41 et MOFFITT, John F., Velázquez, Práctica e idea: estudios dispersos, Málaga, 1991, p. 55-57.

${ }^{25}$ Ibid., p. 109. 
des Cieux, conçue sans péchés, médiatrice entre le monde visible et l'invisible et aussitôt considérée comme une mère mystique, une figure allégorique de l'Église ${ }^{26}$. Au bout du compte, une Marie nécessaire au salut des hommes, envers et contre toutes les assertions de la théologie protestante qui minimisait la grandeur de la mère de Jésus.

Dès les premiers temps du christianisme oriental, l'art des icônes (eikos, image en grec) a relevé le défi de la représentation du divin ${ }^{27}$. Au XVIe siècle, face à la Réforme iconophobe, comment pouvait-on montrer l'illimité, l'intemporel, en un mot l'invisible, sans tomber dans des dévoiements idolâtres? Nous pensons que les tableaux de vision constituent une réponse.

La peinture est vraiment religieuse - dit justement Eric Fuchs - quand elle fait découvrir le lien qui unit la réalité à la présence secrète qui illumine son obscurité [...]. La peinture n'est pas la reproduction fidèle du réel, elle est une infidélité au réel, un refus de ce qui se donne immédiatement à voir, le souci de ce qui est encore caché et qu'il s'agit de dévoiler ${ }^{28}$.

L'origine apostolique de l'Apocalypse a été mise en doute dès les premiers siècles. Ces doutes reparurent avec la Réforme. Dans l'édition de 1522 de sa traduction du Nouveau Testament, Luther s'exprime ainsi : «Bien des choses me manquent dans ce livre et ne me permettent pas de le tenir pour apostolique ni pour prophétique ». Les théologiens luthériens gardèrent cette attitude négative jusque vers le milieu du dix-septième siècle. Le réformateur suisse Ulrich Zwingli déclara, en 1528, à la dispute de Berne, que l'Apocalypse n'était pas un livre biblique. Elle est le seul écrit du Nonveau Testament que Calvin n'ait jamais commenté. Le protestantisme n'a pas développé les conséquences esthétiques possibles de la grâce, occupé qu'il était à défendre une théologie de la parole comprise comme argumentative et rationnelle. L'implication émotionnelle inhérente à l'esthétique de la Contre-Réforme triomphante donne, on le sait, un statut nouveau à l'image. Aussi bien la toile du Gréco que le diptyque de Vélasquez, établit une subtile dialectique entre le visible et l'invisible auquel les deux artistes donnent figure et, ainsi rendent-ils palpable la transcendance. On sait que la vision apocalyptique de Jean est aussi une contestation de l'ordre impérial romain et signifie le triomphe du christianisme sur la Rome des Césars, donc qu'elle s'inscrit dans un contexte idéologique et politique. La présence de la Vierge, portrait peint par l'artiste divin dans le tableau du Gréco ou sous la forme d'un dédoublement dans les toiles de Vélasquez, est bien un signe de la lutte héroïque de la Femme, la propre mère du Christ, aux prises avec les forces du mal. La réforme protestante n'est, aux yeux des Catholiques, que mensonge et illusion et la volonté d'englober toute la réalité de l'existence humaine n'est, pour eux, qu'une tromperie diabolique. L'Incarnation marque la rupture de l'invisibilité

26 Voir STRATTON, Suzanne, «La Inmaculada Concepción en el arte español », Cuadernos de arte e iconografía, Tome I-2, 1998. p. 3-127. Revista virtual de la fundación universitaria española, http:// www.fuesp.com/revistas/pag/cat020:html. «La idea de crear una Real Junta de prelados y teólogos que se ocupara de estudiar todo lo relativo a la Inmaculada Concepción, nació en Sevilla durante los tumultuosos años de 1614 y 1615. Ante las repetidas solicitudes hechas desde Sevilla y ante la insistencia de su tía Sor Margarita de la Cruz de las Descalzas Reales, Felipe III estableció el 2 de Junio de 1616 una Real Junta cuyas funciones serían lograr del papa que acallase a los calumniadores de la doctrina y que definiese la doctrina como dogma ».

27 Voir CUVILLIER, Elian, «La vision comme contestation de l'idole. Apocalypse de Jean et Empire romain », dans J.-M. Marconot et B. Tabuce éds, Iconoclasme et vandalisme. La violence de l'image, Montpellier, Université Montpellier III, 2005, p. 97-103.

${ }^{28}$ FUCHS, Eric, Faire voir l'invisible, réflexions théologiques sur la peinture, Genève, Labor et Fides, 2005, p. 108110. 
totale et définitive de Dieu. Elle se comprend comme la venue salvatrice de l'image du divin dans le monde visible et son unique légitimation théologique. Les représentations picturales de la vision de saint Jean à Patmos deviennent, dans les œuvres d'art analysées, le lieu possible de la révélation. 